des „echten“ Parlamentsbeschlusses..$^{93}$ Der konstitutive Parlamentsbeschluss ermöglicht eine autonome, wesentlich einfachere und schnellere Beteiligung des Bundestages als seine Beteiligung durch Gesetz nach Art. 59 Abs. 2 GG. ${ }^{94}$

93 Dieter Wiefelspütz, a.a.O. (Fn. 2), S. 494. Nach Roman Schmidt-Radefeldt, a.a.O. (Fn. 6), S. 139, stellt der ungeschriebene verfassungsrechtliche Parlamentsvorbehalt einen neuen „Тypus" beteiligungssichernder bindender Parlamentsbeschlüsse dar. Ungenau Peter Dreist, Die Auslandseinsätze der Bundeswehr im Spannungsfeld zwischen Politik und Verfassungsrecht, in: Klaus-Jürgen Bremm / Hans Mack / Martin Rink (Hrsg.), Entschieden für Frieden - 50 Jahre Bundeswehr 1955 bis 2005, Freiburg u.a. 2005, S. 507 (S. 512); ders., in: BWV 2005, S. 29 (S. 34).

94 Gunnar Geiger, in: NZWehrr 2001, S. 133 (S. 146); Juliane Kokott, a.a.O. (Fn. 7), S. 944, S. 948 f.; Hermann Butzer, a.a.O. (Fn. 13), S. 61 (S. 69).

\title{
Die baden-württembergische Landtagswahl vom 26. März 2006: Schwarzes Land mit bunten Tupfern
}

\author{
Oscar W. Gabriel und Kerstin Völkl
}

Bereits in der ersten Umfrage aus Anlass der baden-württembergischen Landtagswahl deutete sich an, dass es auch im Jahr 2006 nicht zu einem Regierungswechsel kommen würde. Mit einem geschätzten Stimmenanteil von 45 Prozent lag die CDU weit vor der SPD. Da die FDP und die Grünen auf jeweils neun Prozent der Wählerstimmen kamen und es keinerlei Diskussion über eine Ampelkoalition gab, schien die Bestätigung der CDU als stärkste Regierungspartei einmal mehr vorprogrammiert ${ }^{1}$. Dennoch warnte die Stuttgarter Zeitung vor einer vereinfachten Sicht der politischen Verhältnisse im Ländle: „Baden-Württemberg gilt als schwarzes Land. Doch ein Streifzug durch die Geschichte der Landtagswahlen gibt den Blick frei auf eine bunt gefleckte politische Landschaft, in

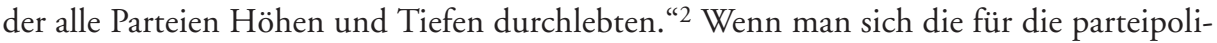
tische Kräfteverteilung im Bund und in den Ländern maßgeblichen Faktoren vor Augen führt, ist die seit 1953 anhaltende Vorherrschaft der CDU in Baden-Württemberg keineswegs so selbstverständlich, wie es scheint: Der Katholikenanteil im Land fällt mit 38 Prozent nicht außergewöhnlich hoch aus, 39 Prozent der erwerbstätigen Bevölkerung sind im produzierenden Gewerbe beschäftigt, ein großer Teil der Bevölkerung lebt in den städtischen Ballungsräumen, darunter allein 2,7 Millionen Einwohner in der Region Stuttgart. Diese Größen sprechen gegen eine stabile Dominanz der CDU. Dennoch

1 Ergebnisse der ersten Wahlumfrage von Infratest-dimap nach Stuttgarter Zeitung (StZ) vom 2. Februar 2006, Internetrecherche, Zugriff am 5. September 2006.

2 Schwarzes Land mit wenig Bunt. Die Wahlen in Baden-Württemberg seit 54 Jahren, in: StZ vom 6. März 2006, Internetrecherche, Zugriff am 5. September 2006. 
scheint es für die SPD unter normalen Umständen fast unmöglich zu sein, die Christdemokraten in der Wählergunst zu überflügeln. Dies zeigte sich auch bei der Landtagswahl im März 2006.

\section{Die Ausgangslage}

Im Vergleich zur vorangegangenen Landtagswahl fand die des Jahres 2006 unter gänzlich anderen bundes- und landespolitischen Vorzeichen statt. Die 2001 auf Bundesebene amtierende rot-grüne Koalition hatte bei der Bundestagswahl 2005 ihre Mehrheit verloren und war von einer Großen Koalition abgelöst worden. Wie die auf der Bundesebene durchgeführten Umfragen zeigten, kam dieser Regierungswechsel der CDU/CSU mehr zugute als der SPD. Die neue Regierungschefin Angela Merkel verfügte über einen starken Kanzlerbonus und war nach dem Ergebnis aller Meinungsumfragen die populärste Bundespolitikerin. Ungeachtet der anhaltend negativen Bewertung der Wirtschaftslage profitierte die Landes-CDU vom Rückenwind aus Berlin.

Auch die landespolitischen Rahmenbedingungen stellten sich gänzlich anders dar als fünf Jahre zuvor. Ein knappes Jahr vor der Landtagswahl war der langjährige Ministerpräsident Erwin Teufel durch den Vorsitzenden der CDU-Landtagsfraktion Günther Oettinger abgelöst worden. Dieser Wechsel war zwar innerparteilich umstritten und in einer Kampfabstimmung zwischen Oettinger und der von Teufel favorisierten Kultusministerin Annette Schavan vollzogen worden. Jedoch bemühte sich die CDU darum, diesen Vorgang als normalen demokratischen Wettbewerb darzustellen, der keine innerparteilichen Gräben hinterlassen hatte. Nach der Übernahme des Ministerpräsidentenamtes hatte Oettinger eine moderate Kabinettsumbildung vorgenommen, bei der mit Sozialminister Andreas Renner und Landwirtschaftsminister Peter Hauk lediglich zwei seiner Vertrauten ins Kabinett aufgenommen wurden, alle anderen von seinem Vorgänger berufenen Minister aber ihre Ämter behielten. In inhaltlicher Hinsicht setzte die neue Regierung eher auf Kontinuität als auf Wandel, was in Anbetracht der kurzen Zeit zwischen der Kabinettsumbildung und dem Wahlkampfauftakt auch kaum anders möglich gewesen wäre. Lediglich das der Öffentlichkeit im Oktober vorgestellte Programm „Ganztagsbetreuung für Kinder" setzte einen neuen Akzent. Es war aber nicht als grundlegende Neuorientierung der Regierungspolitik zu werten. Auch wenn der neue Ministerpräsident nicht über den Amtsbonus seines Vorgängers verfügte, hatte der Wechsel im Amt per Saldo die ohnehin schwierige Ausgangslage der SPD nicht erleichtert. Die 2001 mit einem gewissen Erfolg praktizierte Strategie, die Wahlentscheidung auf die Alternative zwischen einem alten, verbrauchten Amtsinhaber und einer neuen, jungen und innovativen Oppositionskandidatin zuzuspitzen, hatte keine reale Grundlage.

Die anscheinend problemlose Kabinettsumbildung fand ihren Niederschlag in guten Umfragedaten. 58 Prozent der Befragten waren mit der Arbeit der Landesregierung zufrieden (2001: 66 Prozent). Die weit verbreitete Wechselstimmung, die sich 2001 gezeigt hatte, war 2006 nicht einmal im Ansatz erkennbar. Während sich im Februar 2001 eine leichte Mehrheit der Befragten für einen Regierungswechsel ausgesprochen hatte (47 zu 
45 Prozent), gaben 200660 Prozent einer Fortsetzung der amtierenden Regierungskoalition den Vorzug 3 .

Die Mehrheit der Befragten (57 Prozent) schätzte auch die Wirtschaftslage BadenWürttembergs positiv ein, allerdings stellt sich diese Bewertung in Abhängigkeit von der gewählten Vergleichsperspektive sehr unterschiedlich dar. Verglichen mit 2001 (80 Prozent positive Bewertungen) hatte sich die Stimmungslage drastisch verschlechtert. Im Vergleich mit Rheinland-Pfalz und Sachsen-Anhalt, wo am selben Tag Wahlen stattfanden $^{4}$, waren die baden-württembergischen Wähler allerdings wesentlich positiver gestimmt. Insgesamt war die Ausgangslage für die CDU zu Beginn des Wahlkampfes 2006 zumindest nicht schwieriger als 2001. Sie profitierte von den positiven bundespolitischen Rahmenbedingungen, hatte einen schwierigen Wechsel im Amt des Regierungschefs erfolgreich bewältigt, operierte in einem vergleichsweise positiven wirtschaftlichen Klima und schien sowohl für die FDP als auch für die Grünen als Regierungspartner in Frage zu kommen.

\section{Der Wahlkampf}

Die spannungsarme Ausgangslage fand ihren Niederschlag im Wahlkampf aller im Landtag vertretenen Parteien. Die frühe Phase war in der Öffentlichkeit kaum wahrnehmbar. Auch die Medienberichterstattung fiel zunächst sehr zurückhaltend aus. Die Plakatierung war spärlich, Fernsehduelle und Kundgebungen waren gänzlich in die heiße Wahlkampfphase verlagert worden. Diese begann am 1. März 2006 mit Großkundgebungen der vier Landtagsparteien. Während die CDU ihre Auftaktveranstaltung ausschließlich mit Ministerpräsident Oettinger und CDU-Generalsekretär Thomas Strobl bestritt, boten die anderen Parteien neben ihren landespolitischen Führungsfiguren prominente Bundespolitiker wie Franz Müntefering (SPD), Reinhard Bütikofer und Fritz Kuhn (Grüne) sowie Dirk Niebel (FDP) auf5.

Die Nominierung der Spitzenkandidaten der vier Landtagsparteien war bereits lange zuvor erfolgt ${ }^{6}$. In der CDU war es unumstritten, dass Ministerpräsident Oettinger nach seinem Erfolg im innerparteilichen Machtkampf die CDU in die Wahl führen würde. Wie bereits 2001 hatte die SPD die Landesvorsitzende Ute Vogt zur Spitzenkandidatin nominiert. Ihre Chancen, das gute Ergebnis des Jahres $2001 \mathrm{zu}$ wiederholen oder gar zu verbessern, wurden von vornherein als gering veranschlagt. Vogt hatte zwar im Vorfeld des Wahlkampfes ihre Funktion als Parlamentarische Staatssekretärin in Berlin aufgegeben, dadurch aber kaum das Manko einer geringen Präsenz in der Landespolitik wettmachen können. Auch ihre Rolle beim Sturz des SPD-Bundesvorsitzenden Müntefering hat-

3 Die Angaben beziehen sich auf die von Infratest dimap erhobenen Umfragedaten.

4 Vgl. zu diesen Landtagswahlen die Beiträge von Heiko Gothe und Everhard Holtmann in diesem Heft der ZParl.

5 Vgl. Politischer Aschermittwoch - Landauf, landab Geplänkel. Der Wahlkampf kommt im Südwesten nur langsam auf Touren, in: StZ vom 2. März 2006, Internetrecherche, Zugriff am 5. September 2006.

6 Vgl. Landtagswahl wird Bewährungsprobe. Nach elf Monaten als Nachfolger Teufels: Ministerpräsident Oettinger ringt um Vertrauen, in: StZ vom 28. Februar 2006, Internetrecherche, Zugriff am 5. September 2006. 
te sie in der Partei und in der Öffentlichkeit Sympathien gekostet. Hinzu kamen Ungeschicklichkeiten im Verlauf des Wahlkampfes. Im Vergleich zu 2001 hatte die von der SPD angebotene personelle Alternative zum Ministerpräsidenten deutlich an Zugkraft eingebüßt.

Die beiden kleinen Landtagsparteien, die FDP und die Grünen, mussten den Wahlkampf mit neuen Spitzenkandidaten bestreiten. An Stelle ihres langjährigen Landesvorsitzenden Walter Döring, der in Folge einer Verwicklung in eine Spendenaffäre sein Amt als Wirtschaftsminister aufgegeben hatte, trat Justizminister Ulrich Goll als Spitzenkandidat der FDP an. Die Grünen hatten nach der Wahl von Dieter Salomon zum Freiburger Oberbürgermeister Winfried Kretschmann zum Fraktionsvorsitzenden und zum Spitzenkandidaten für die Landtagswahl bestellt.

Wenig Brisanz ließ auch die Auseinandersetzung über politische Sachfragen erkennen. Zum Teil war dies darauf zurückzuführen, dass die Hauptkontrahenten im Lande, die CDU und die SPD, seit dem Herbst auf der Bundesebene gemeinsam regierten. Einer scharfen Polarisierung waren damit Grenzen gesetzt. In den großen Wahlkampfthemen, der Verbesserung der Arbeitsmarktlage und der Ankurbelung der Wirtschaft, waren keine grundlegenden Kontroversen erkennbar. Die CDU vertrat die Position, in diesen und anderen Bereichen eine gute Politik gemacht zu haben und insofern keine prinzipielle Neuorientierung vornehmen zu müssen. Als Schwerpunkt seiner Regierungsarbeit kündigte Oettinger im ersten TV-Duell mit Ute Vogt den Ausbau Baden-Württembergs zu einem Kinderland sowie Maßnahmen zur Verbesserung der Chancen älterer Arbeitnehmer auf dem Arbeitsmarkt an. Mit den Forderungen nach einer verstärkten Förderung arbeitsloser Jugendlicher, einer intensiveren Sprachförderung von Migrantenkindern in den Schulen und Kindergärten und einem Verkauf von Landesbeteiligungen formulierte die SPD kein Kontrastprogramm zur Regierungspolitik, sondern setzte lediglich etwas andere Akzente. Anders als mit der Bildungspolitik im Wahlkampf 2001 gelang es der SPD diesmal nicht, ein Thema zu besetzen und es mit der Person der Spitzenkandidatin zu verknüpfen. Dissens zwischen den beiden großen Parteien sowie FDP und Grünen bestand in der Bewertung der gentechnikfreien Landwirtschaft und der Verlängerung der Laufzeit von Kernkraftwerken, die Oettinger und die Regierungsparteien befürworteten, die Oppositionsparteien aber entschieden ablehnten ${ }^{7}$. Da diese Frage im Wahlkampf und auch in den Einschätzungen der Wählerschaft eine untergeordnete Rolle spielte, fehlte der Stoff, aus dem polarisierende und mobilisierende Wahlkämpfe gemacht sind.

Zu den wenigen Spannungsmomenten der Wahlkampfzeit gehörte die Koalitionsfrage. Zwar bezeichneten CDU und FDP ihr seit 1996 bestehendes Regierungsbündnis übereinstimmend als erfolgreich, jedoch vermied die CDU im Gegensatz zu ihrem Partner eine klare Koalitionsaussage. Die Sympathie von Ministerpräsident Oettinger für ein

7 Vgl. TV-Duell - Schlagabtausch vor der Kamera. Laut einer Umfrage kann die CDU im Land auf eine absolute Mehrheit hoffen, in: StZ vom 2. März 2006; vgl. auch Interview - „Ich will Vertrauen stärken“. Günther Oettinger (CDU) setzt auf alle Gesellschaftsgruppen, in: StZ vom 14. März 2006; Interview - „Die Lage verlangt mehr Ernst“. Ute Vogt (SPD) will Politik an den Menschen orientieren, in: StZ vom 9. März 2006.; Letzte TV-Debatte - Streit über den Streik. Aussagen zu möglichen Bündnissen vage, in: StZ vom 23. März 2006; Internetrecherche, Zugriff jeweils am 5. September 2006. 
schwarz-grünes Bündnis war bekannt, hatte er doch angeblich schon vor fünf Jahren eine derartige Zusammenarbeit favorisiert, seine Präferenzen aber nicht gegen den damaligen Ministerpräsidenten durchsetzen können. Da auch die Grünen, insbesondere ihr Spitzenkandidat Kretschmann, eine Koalition nicht ausschlossen, lag die Bildung einer schwarz-grünen Regierung nicht jenseits des Vorstellbaren. Jedoch sprachen vor allem zwei Gründe gegen diese Option: Eine schwarz-grüne Zusammenarbeit würde für die CDU konflikthaltiger sein als ein Weiterregieren mit der FDP, die sich innerhalb der Regierung wenig profiliert hatte. Zudem war sowohl in der CDU- als auch in der Grünen-Anhängerschaft mit Widerständen gegen eine solche Koalition zu rechnen. Da sich während des Wahlkampfes wiederholt zeigte, dass die Gräben innerhalb der CDU tiefer waren, als es deren Repräsentanten zugaben, wäre ein offenes Eintreten Oettingers für ein Bündnis mit den Grünen eine riskante Strategie gewesen. Dem standen gute Gründe für ein Zusammengehen gegenüber: In etlichen Kommunen des Landes, nicht zuletzt in der Landeshauptstadt Stuttgart, hatte sich die Zusammenarbeit der beiden Parteien als konstruktiv erwiesen und eine Wiederwahl des CDU-Oberbürgermeisters Wolfgang Schuster ermöglicht ${ }^{8}$. Außerdem sahen etliche Politiker und Mitglieder von CDU und Grünen in einem solchen Bündnis eine Chance, eine innovativere Politik zu betreiben, die Optionen ihrer Parteien im Parteienwettbewerb zu erweitern und ein Signal nach Berlin zu senden. Zwar gab es in der Energie-, Verbraucherschutz- und Ausländerpolitik Gegensätze, in vielen Fragen der Haushalts- und Wirtschaftspolitik bestanden jedoch große Gemeinsamkeiten. Die SPD äußerte sich nicht klar zur Koalitionsfrage9 , was angesichts der geringen Chancen für einen Regierungswechsel nicht verwunderlich war.

Die Spannungsarmut des Wahlkampfes war schließlich auch an einigen, hingebungsvoll von der Regenbogenpresse breitgetretenen Ereignissen erkennbar ${ }^{10}$, und sie fand ihren Niederschlag in den während des Wahlkampfes veröffentlichten Umfragen. Selbst in

8 Der Kandidat der Grünen hatte sich nach dem ersten Wahlgang aus dem Rennen um das Amt des Oberbürgermeisters zurückgezogen und dies mit einer Wahlempfehlung zu Gunsten des CDU-Kandidaten verbunden.

9 Letzte TV-Debatte - Streit über den Streik. Aussagen zu möglichen Bündnissen vage, in: StZ vom 23. März 2006, Internetrecherche, Zugriff am 5. September 2006.

10 Die vier herausragenden Meldungen waren die folgenden: Sozialminister Andreas Renner (CDU) war nach einer im Juli 2004 mit dem katholischen Bischof Gebhard Fürst geführten Auseinandersetzung und heftiger innerparteilicher Kritik von seinem Amt zurückgetreten. Angeblich soll er im Sommer 2004 in einem Disput über seine Schirmherrschaft für die Schwulenparade „Christopher Street Day“ zu Fürst gesagt haben: „Halten Sie sich da raus. Fangen Sie doch erst einmal damit an, Kinder zu zeugen" (Die Welt vom 28. Januar 2006, Internetrecherche, Zugriff am 15. September 2006). Weiteren Stoff für die Skandalpresse lieferte Staatsminister Willi Stächele (CDU), der auf der Rückfahrt von einem Empfang seine Ehefrau an einer Auto-Rastanlage vergessen hatte (vgl. Netzeitung.de vom 3. März 2006, Internetrecherche, Zugriff am 15. September 2006). Ute Vogt (SPD) dürfte sich den größten Patzer geleistet haben. Sie hatte in einem Interview mit dem privaten Sender Hitradio Antenne 1 drei Tage vor der Wahl zugegeben, schon einmal einen Orgasmus vorgetäuscht zu haben. Die Redaktion vermarktete die Geschichte gezielt. Bild titelte tags darauf auf Seite eins „Erste Politikerin beichtete Orgasmus-Lüge“ (vgl. TAZ vom 24. März 2006, Internetrecherche, Zugriff am 15. September 2006). In der gleichen Sendereihe hatte Winfried Kretschmann (Grüne) eingestanden, schon einmal Marihuana geraucht und 18 Liter Bier an einem Abend in seiner Studentenzeit getrunken zu haben (vgl. Hit-Radio Antenne 1 vom 20. März 2006, Internetrecherche, Zugriff am 15. September 2006). 
der Woche vor der Wahl erklärten nur 56 Prozent der Befragten, sich dafür zu interessieren. Da dies nur sechs Prozentpunkte mehr als zu Beginn der heißen Wahlkampfphase waren $^{11}$, konnte von einer wahlkampfbedingten Wählermobilisierung nicht die Rede sein. Eine Wechselstimmung war nicht erkennbar, und in allen Umfragen sowie bei sämtlichen für den Wahlentscheid maßgeblichen Einstellungen lag die CDU mit weitem Abstand vor der SPD. Seit der Publikation der letzten Umfragedaten drehte sich die öffentliche Diskussion nur noch um die Frage, ob es der CDU gelingen würde, die absolute Mehrheit der Mandate zu gewinnen, und falls nicht, ob sie die bestehende Koalition fortsetzen oder durch ein schwarz-grünes Bündnis ablösen würde.

\section{Das Wahlergebnis}

\subsection{Wahlbeteiligung und Stimmenverteilung im Land}

Wie bereits der Wahlkampf hielt auch das Ergebnis der Landtagswahl wenig Überraschendes bereit. Die große Ausnahme stellte die Wahlbeteiligung dar. Sie war nach dem niedrigen Stand des Jahres 2001 erneut deutlich gefallen, nämlich um 9,2 Prozentpunkte. Damit war sie so niedrig wie noch nie in der Geschichte des Landes und hatte selbst den historischen Tiefstwert von 59,0 Prozent (1960) noch deutlich unterboten. Zudem setzte sich der 1972 einsetzende Trend einer sinkenden Wahlbeteiligung fort. In absoluten Zahlen ausgedrückt waren mehr als eine halbe Million Bürger im Vergleich zu vor fünf Jahren der Wahlurne fern geblieben. Lediglich 53,4 Prozent der Wahlberechtigten gaben ihre Stimme ab (vgl. Tabelle 1). Dabei sank die Wahlbeteiligung gegenüber 2001 in allen Wahlkreisen des Landes. Im badischen Landesteil sowie in den Großstädten fiel sie besonders niedrig aus ${ }^{12}$.

Die prozentuale Stimmenverteilung auf die Parteien entsprach dagegen weitgehend den Erwartungen und den zuvor veröffentlichten Umfragewerten. Mit 44,2 Prozent der gültigen Stimmen verfehlte die CDU nur knapp die absolute Mehrheit der Landtagsmandate und erzielte nahezu das gleiche Ergebnis wie 2001 (vgl. Tabelle 1). Auf Grund der niedrigen Wahlbeteiligung verloren die Christdemokraten allerdings im Vergleich zu 2001 über 280.000 Wähler. Drastische Verluste hatte die SPD zu verzeichnen. Sie erreichte 2006 lediglich 25,2 Prozent und musste Stimmeneinbußen von über einer halben Million Wählern hinnehmen. Nach 1996 war dies für die Sozialdemokraten das schlechteste Resultat bei einer Landtagswahl in Baden-Württemberg ${ }^{13}$. Von ihren Verlusten profitierten insbesondere die kleinen Parteien. Beachtliche Zugewinne konnten die Grünen verbuchen. Mit einem Stimmenanteil von 11,7 Prozent verfehlten sie nur knapp ihr bisher bestes Landtagswahlergebnis in Baden-Württemberg (1996: 12,1 Prozent). Es gelang ihnen, ihre Anhängerschaft von rund 350.000 auf knapp 463.000 Wähler zu vergrößern.

11 Infratest dimap, Umfragen vom 30. Januar bis 1. Februar und vom 13. bis 15. März 2006. 12 Vgl. Statistisches Landesamt Baden-Württemberg 2006, Wahl zum 14. Landtag von BadenWürttemberg am 26. März 2006. Vorläufige Ergebnisse.

13 Vgl. zur Wahl 1996: Roland Sturm, Die baden-württembergische Landtagswahl vom 24. März 1996: Normalisierung oder kleinster gemeinsamer Nenner?, in: ZParl, 27. Jg. (1996), H. 4, S. $602 \mathrm{ff}$. 


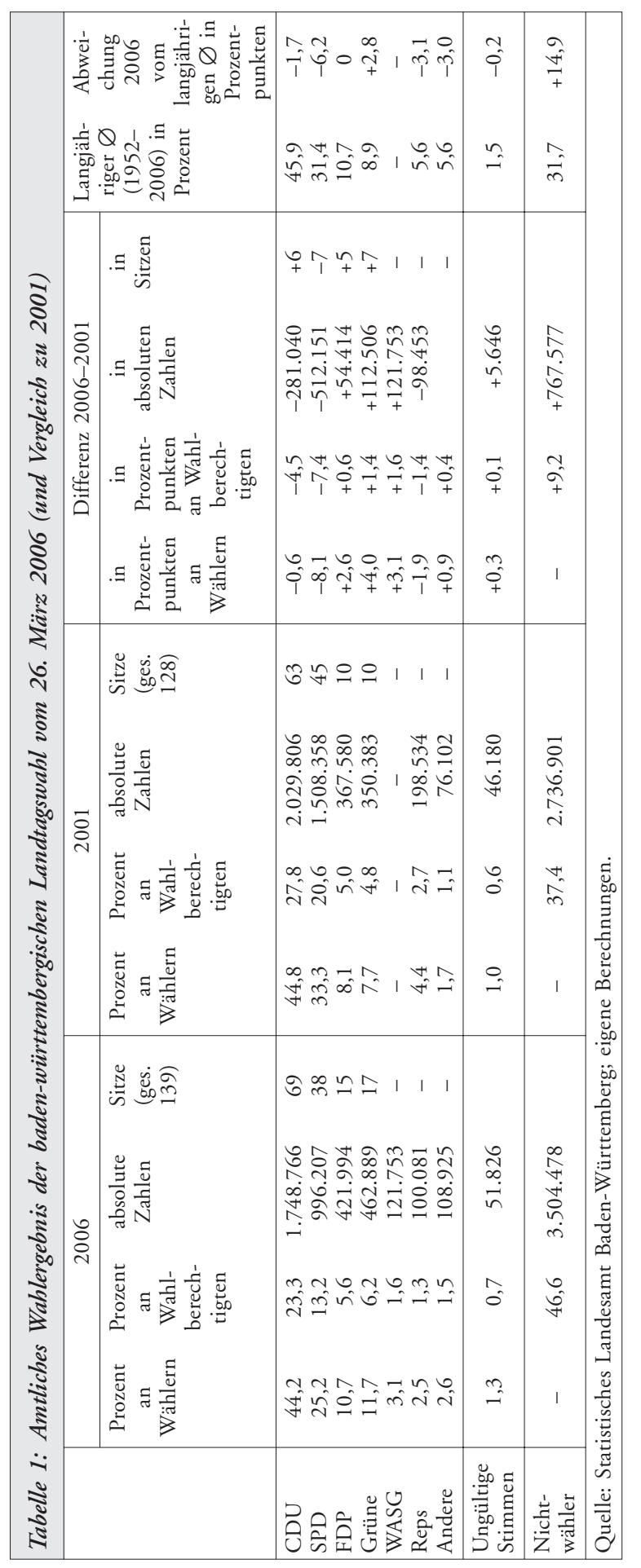


Damit sind die Grünen diejenige Partei, die ihren Rückhalt unter den baden-württembergischen Bürgern trotz rückläufiger Wählerzahlen am deutlichsten ausbauen konnte. Die FDP erhielt 10,7 Prozent der gültigen Stimmen und erzielte damit erstmals seit 1968 wieder ein zweistelliges Wahlergebnis. Wie die Grünen konnten auch die Liberalen neue Wähler gewinnen (54.000 Stimmen). Trotz dieses guten Abschneidens mussten sie die 2001 nach über 15 Jahren zurückeroberte Position der drittstärksten Fraktion im Landtag wieder an die Grünen abtreten. Einen Achtungserfolg verzeichnete die erstmals bei einer Landtagswahl in Baden-Württemberg angetretene WASG. Zwar scheiterte sie mit 3,1 Prozent der Wählerstimmen klar an der Fünf-Prozent-Hürde, jedoch hatte sie mit knapp 122.000 Stimmen den größten Zugewinn. Die Republikaner verpassten nach 2001 auch 2006 mit 1,5 Prozent der Stimmen den Einzug in den Landtag.

Auf Grund der niedrigen Wahlbeteiligung verdeckt das amtliche Endergebnis, bei dem die Stimmenanteile der Parteien auf die Anzahl der Wähler prozentuiert werden, das tatsächliche Ausmaß der Wählerbewegung. Wählt man stattdessen als Prozentuierungsbasis der Parteianteile die Zahl der Wablberechtigten, dann bilden die Werte nicht nur die Wählerwanderungen zwischen den Parteien ab, sondern auch die Verschiebungen zwischen dem Lager der Wähler und dem der Nichtwähler. Demnach kam die CDU nur auf knapp ein Viertel der Stimmen aller Wahlberechtigten und verlor im Vergleich zu 2001 4,5 Prozentpunkte. Die SPD konnte nur noch gut 13 Prozent der Stimmen der Wahlberechtigten auf sich vereinigen und büßte gegenüber 2001 sieben Prozentpunkte ein. Wenn man sich vor Augen führt, dass die Wählerausschöpfungsquoten der FDP und der Grünen etwas über fünf Prozent lagen, werden die Integrations- und Mobilisierungsschwächen der demokratischen Parteien vollends deutlich. Sie konnten lediglich 48 Prozent der Wahlberechtigten an sich binden. Im langfristigen Durchschnitt fallen ebenfalls die hohe Zahl der Nichtwähler und das schlechte Abschneiden der SPD auf. Nie zuvor waren so wenig Wahlberechtigte der Aufforderung nachgekommen, einen neuen Landtag zu wählen, und keine andere Partei musste solche starken kurzfristigen Stimmenverluste hinnehmen wie die SPD. Auch die Republikaner schnitten schlechter als im langfristigen Durchschnitt ab. Die CDU lag nur geringfügig unter ihrem langfristigen Mittel. Ein überdurchschnittliches Resultat erzielten lediglich die Grünen, während die FDP in langfristiger Perspektive im Rahmen ihrer Möglichkeiten blieb.

\subsection{Das Ergebnis in den Wahlkreisen}

Der Vorsprung der CDU vor der SPD bei der Landtagswahl 2006 spiegelt sich nicht nur in der Stimmenverteilung, sondern auch in der Anzahl der Erstmandate wider. Die Christdemokraten gewannen 69 der 70 Wahlkreise. Während die CDU fünf Jahre zuvor in 63 der 70 Wahlkreise vor der SPD gelegen hatte, gelang es den Sozialdemokraten 2006 nur im Wahlkreis Mannheim I, das Direktmandat zu erringen. Die CDU verbuchte in 28 der 70 Wahlkreise Stimmengewinne und musste in 40 Wahlkreisen Verluste hinnehmen. Ihre Hochburgen hatte die Partei traditionsgemäß im ländlichen Raum mit einer niedrigen Bevölkerungsdichte sowie in Gegenden mit einem hohen Katholikenanteil. Überdurchschnittliche Zuwächse verzeichnete sie in den SPD-Diasporagebieten und 
Wahlkreisen mit einem hohen Protestantenantei ${ }^{14}$. Ausgesprochen hart traf es die Sozialdemokraten, die in allen 70 Wahlkreisen Verluste hinnehmen und sich viermal sogar mit dem dritten Platz begnügen mussten. In Biberach, Freiburg I und Tübingen wurden sie von den Grünen und in Freudenstadt von der FDP überholt. Ihre besten Ergebnisse erzielte die SPD in urban geprägten Wahlkreisen mit einer hohen Bevölkerungsdichte und Erwerbslosenquote sowie in Wahlkreisen mit einem hohen Anteil Beschäftigter im Dienstleistungsgewerbe. Dort mussten sie allerdings überdurchschnittlich große Verluste hinnehmen, während diese in Wahlkreisen mit einem starken produzierenden Gewerbe geringer ausfielen als im Landesdurchschnitt.

Im Vergleich zur Landtagswahl 2001 erzielte die FDP in 65 der 70 Wahlkreise Stimmengewinne. Besonders erfolgreich waren die Liberalen in protestantisch geprägten Gebieten sowie in Wahlkreisen mit einer hohen Kaufkraft. Deutliche Zuwachsraten verzeichneten sie in SPD-Diasporagebieten und Wahlkreisen mit hohem Katholikenanteil. Im Einklang mit ihrer von den Liberalen zurückeroberten Position als drittstärkster Kraft im Landtag erhielten die Grünen in 36 der 70 Wahlkreise mehr Wählerstimmen als die FDP und verbuchten in allen 70 Wahlkreisen Gewinne. Ihre besten Ergebnisse erzielten sie traditionsgemäß in den Universitätsstädten und Wahlkreisen mit einem hohen Akademikeranteil sowie solchen mit einem starken Dienstleistungssektor. Dort sowie in den CDU-Hochburgen waren ihre Stimmengewinne zugleich überdurchschnittlich groß. In Wahlkreisen mit einem hohen Arbeiteranteil blieben sie unter dem Landesdurchschnitt. Auch wenn die WASG auf Landesebene an der Fünf-Prozent-Hürde scheiterte, gelang es ihr immerhin in vier Wahlkreisen, diese zu überspringen ${ }^{15}$.

\subsection{Bestimmungsfaktoren des Wählervotums}

Unter Wirkungsgesichtspunkten unterscheidet man in der empirischen Wahlforschung zwischen zwei Arten von Erklärungsfaktoren der Parteipräferenz: Als langfristig stabile Einflussgröße bestimmt die Parteiidentifikation über die Verteilung der Stammwähler. Daneben gibt es mit der Themen- und Kandidatenorientierung kurzfristig wirksame Faktoren, die sich definitionsgemäß von Wahl zu Wahl ändern können und zu entsprechenden Abweichungen der Wahlergebnisse von der langfristigen parteipolitischen Kräfteverteilung führen ${ }^{16}$. Außerdem müssen bei Landtagswahlen bundespolitische Faktoren berücksichtigt werden. Da die Wahlentscheidung bei der baden-württembergischen Landtagswahl 2006 stark von landespolitischen Themen geprägt war, kann der Einfluss

14 Hochburgen beziehungsweise Diasporagebiete einer Partei sind jeweils die zehn Wahlkreise, in denen diese Partei die höchsten beziehungsweise die niedrigsten Wählerstimmen erreicht hat.

15 Vgl. Statistisches Landesamt Baden-Württemberg 2006, a.a.O.

16 Vgl. Angus Campbell / Philip E. Converse / Warren E. Miller / Donald E. Stokes, The American Voter, New York u.a. 1960; Oscar W. Gabriel, Parteiidentifikation, Kandidaten und politische Sachfragen als Bestimmungsfaktoren des Parteienwettbewerbs, in: ders. / Oskar Niedermayer / Richard Stöss (Hrsg.), Parteiendemokratie in Deutschland, Wiesbaden 2001, S. 228 - 249; ausführlicher: Harald Schoen / Cornelia Weins, Der sozialpsychologische Ansatz zur Erklärung von Wahlverhalten, in: Jürgen W. Falter / Harald Schoen (Hrsg.), Handbuch Wahlforschung. Ein einführendes Handbuch, Wiesbaden 2005, S. 187 - 242. 
der Bundespolitik bei der folgenden Analyse vernachlässigt werden ${ }^{17}$. Folglich bezieht sich die Untersuchung auf die drei Kernvariablen des so genannten sozialpsychologischen Erklärungsmodells des Wählerverhaltens. Dabei ist die Parteiidentifikation im Unterschied zu den Themen- und Kandidatenorientierungen keine genuin auf die Landespolitik bezogene Einflussgröße.

Der wichtigste Bestimmungsfaktor der Parteipräferenz ist die Parteiidentifikation. In der letzten vor dem Wahltag durchgeführten Umfrage der Forschungsgruppe Wahlen (FGW) gaben drei Viertel der Befragten an, über eine Parteiidentifikation zu verfügen ${ }^{18}$. Ein Drittel fühlte sich der CDU verbunden (34 Prozent), lediglich 21 Prozent der SPD, acht Prozent entfielen auf die Grünen, vier Prozent auf die FDP. Im Vergleich mit dem Jahr 2001 hat sich der Anteil der Parteiidentifizierer erwartungsgemäß kaum verändert. In dieser Verteilung der Parteiidentifikation, die sich keineswegs zwingend aus der Sozialstruktur des Landes ergibt, liegt die wichtigste Erklärung der langfristigen, insbesondere seit dem Beginn der 1970er Jahre klaren Dominanz der CDU in Baden-Württemberg. Um aus Wahlen als stärkste Partei hervorgehen zu können, reicht es für die Christdemokraten aus, ihre Stammwähler zu mobilisieren. Dies gilt in der Regel selbst dann noch, wenn sie über die Kompetenzbewertung und die Einstellung zum Spitzenkandidaten nur wenige Wähler an sich binden können. In ganz einfachen Worten ausgedrückt wäre die CDU in Baden-Württemberg bei der derzeitigen Verteilung der Parteiidentifikation selbst mit einem schwachen Spitzenkandidaten, wenig überzeugenden programmatischen Angeboten und einer mageren Leistungsbilanz der Landesregierung von der SPD nur schwer zu schlagen. Auch wenn es den Sozialdemokraten gelänge, durch einen attraktiven Kandidaten und überzeugende politische Konzepte die CDU aus der Position der stärksten Partei zu verdrängen, müsste sie ihre Stimmengewinne in erster Linie durch einen Einbruch in das Lager der CDU oder der FDP erzielen. Im Falle einer deutlichen Schwächung der Grünen, die zudem zur Bildung einer rot-grünen Mehrheit zur Verfügung stehen müssten, würde möglicherweise selbst der Gewinn der relativen Mehrheit der Landtagsmandate nicht dafür ausreichen, den Ministerpräsidenten zu stellen. Die partielle Entkoppelung von Sozialstruktur und Parteiidentifikation, die sich vor allem im württembergischen Landesteil zeigt, ist ein wahlsoziologisch außerordentlich interessanter, bisher kaum diskutierter Aspekt des politischen Lebens im Südwesten.

Die langfristig wirksame Verteilung der Parteiidentifikation bildete den Sockel des Wahlerfolges der CDU, kann ihn aber allein ebenso wenig erklären wie das Wahldesaster der SPD. Themen- und Kandidatenorientierungen spielten in mehrerlei Hinsicht eine wichtige Rolle für die Wahlentscheidung der Bürger. Zunächst fühlten sich 32 Prozent

17 Analysen, die außer der Parteibindung, der Themen- und Kandidatenorientierung den Einfluss der Bundespolitik bei der baden-württembergischen Landtagswahl 2006 berücksichtigten, brachten keinen zusätzlichen Erklärungswert. Daraus kann geschlussfolgert werden, dass die Bundespolitik für die Wahlentscheidung der Bürger in Baden-Württemberg weitgehend irrelevant war. Außerdem liegen Umfragedaten vor, nach denen die Wähler für ihre Entscheidung primär landespolitische Faktoren heranzogen, während die Stimmenthaltung in erster Linie bundespolitisch motiviert war. Vgl. hierzu Oscar W. Gabriel / Kerstin Völkl, Wählerverhalten bei der Landtagswahl 2006 in Baden-Württemberg, in: Josef Schmid / Udo Zolleis (Hrsg.), Wahlen im Südwesten, Münster 2007 (im Erscheinen).

18 Alle präsentierten Ergebnisse basieren auf der von der Forschungsgruppe Wahlen durchgeführten Vorwahlerhebung. 
der Wahlberechtigten keiner Partei verbunden. Sofern diese Bürger sich zur Teilnahme an der Wahl entschließen, hängt ihre Entscheidung ausschließlich von kurzfristig wirksamen Faktoren ab. Hinzu kommen Personen mit einer schwachen Parteiidentifikation, die ebenfalls ihre Themen- und Kandidatenorientierungen in den Wahlentscheid einfließen lassen.

Im Einklang mit dem schon lange auf der Bundes- und Landesebene beobachtbaren Trend hielten die Baden-Württemberger die Arbeitslosigkeit beziehungsweise die gegenwärtige Situation bei Arbeits- und Ausbildungsplätzen für das wichtigste Problem in ihrem Land. Damit nahm dieses Thema wie schon 2001 die Spitzenposition auf der politischen Agenda ein, jedoch hatte sich die Zahl derjenigen, die ihm die größte Relevanz zusprachen, fast verdoppelt (30 beziehungsweise 57 Prozent). Während das Thema Schule und Bildung bei der Landtagswahl 2001 mit 20 Prozent unmittelbar hinter der Lage auf dem Arbeitsmarkt rangierte, lag es 2006 mit neun Prozent weit abgeschlagen auf Platz zwei. Andere Themen wie die Familienpolitik sowie der zum Zeitpunkt der Wahl aktuelle Tarifstreit und Müllstreik wurden nur von einer Minderheit der Wähler als relevant eingestuft.

Da die Bürger die Problemlösungskompetenz in den Bereichen Arbeit und Bildung traditionell sozialdemokratischen Parteien zuordnen, könnte man annehmen, dass sich die SPD aufgrund der Struktur der politischen Agenda gegenüber der CDU im Vorteil befand. Tatsächlich stellte sich die Situation anders dar: Mehr als die Hälfte der Bürger trauten den Christdemokraten am ehesten die Schaffung neuer Arbeitsplätze sowie die Lösung der wirtschaftlichen und finanziellen Probleme des Landes zu. Gegenüber 2001 vergrößerte sich damit der Abstand zwischen CDU und SPD deutlich zu Gunsten der Christdemokraten. Allerdings ordnete ein Viertel der Befragten keiner Partei die Kompetenz zur Lösung wirtschafts- und arbeitsmarktpolitischer Probleme zu (vgl. Tabelle 2). Eine Erklärung des Kompetenzvorsprunges der CDU liegt in der im Bundesvergleich überdurchschnittlich guten Beschäftigungslage und Prosperität Baden-Württembergs, welche die Wähler auch entsprechend wahrnahmen. Dies zeigt sich vor allem im Vergleich mit den beiden Bundesländern, in denen am 26. März 2006 ebenfalls Landtagswahlen stattfanden und in denen die Wirtschaftslage deutlich negativer bewertet wurde als in Baden-Württemberg. Weitere Domänen der CDU waren die Verkehrs- und die Ausländerpolitik.

Bei dem zweitwichtigsten Problem, der Schul- und Bildungspolitik, waren die Bürger in der Bewertung der Fähigkeiten der beiden großen Volksparteien gespalten. Der eine Teil traute der CDU die bessere Politik zu, der andere eher der SPD. Damit büßten die Sozialdemokraten ihren im Jahr 2001 erworbenen Kompetenzvorsprung vor der CDU ein. Fünf Jahre zuvor war es den Sozialdemokraten im Wahlkampf gelungen, die Aufmerksamkeit der Bürger auf die Schwächen der Regierung in der Bildungspolitik zu lenken und ihre eigene Problemlösungskompetenz mit der Person Ute Vogts zu verbinden. 2006 hatte das Thema Bildung für die SPD seine Schubkraft verloren, weil die Christdemokraten ihre 2001 gegebenen Wahlversprechen weitgehend eingelöst und der SPDKritik an der Regierung die Grundlage entzogen hatten. So wurden 5.500 neue Lehrer eingestellt, um dem im Wahlkampf von der SPD scharf kritisierten Ausfall von Unter- 


\begin{tabular}{|c|c|c|c|c|}
\hline & $\mathrm{CDU}$ & SPD & keine & $\mathrm{N}$ \\
\hline Lösung wirtschaftlicher Probleme & 55 & 14 & 24 & 1293 \\
\hline Schaffung neuer Arbeitsplätze & 47 & 15 & 29 & 1299 \\
\hline Lösung der Finanzprobleme & 50 & 14 & 28 & 1268 \\
\hline Schul- und Bildungspolitik & 39 & 38 & 9 & 1210 \\
\hline Familienpolitik & 34 & 43 & 9 & 1264 \\
\hline Vereinbarkeit von Beruf und Familie & 30 & 43 & 10 & 1198 \\
\hline Ausländerpolitik & 43 & 25 & 10 & 1165 \\
\hline Verkehrspolitik & 53 & 17 & 11 & 1013 \\
\hline
\end{tabular}

richtstunden an den Schulen entgegenzuwirken ${ }^{19}$. Auch das vergleichsweise gute Abschneiden Baden-Württembergs bei der PISA-Studie könnte die Zuweisung bildungspolitischer Kompetenz an die CDU begünstigt haben.

Der einzige Bereich, in dem die Wähler den Sozialdemokraten mehr zutrauten als der CDU, war die Familienpolitik und das damit eng verbundene Thema der Vereinbarkeit von Familie und Beruf. Die intensiven Bemühungen der CDU im Vorfeld der Wahl, auf diesem Gebiet Profil zu gewinnen, scheinen bei den Wählern keine Resonanz gefunden zu haben. Hierfür dürften vor allem zwei Gründe verantwortlich gewesen sein. Zwar setzte die CDU-geführte Landesregierung in der vorherigen Legislaturperiode einige Vorhaben zur Verbesserung der Betreuungsmöglichkeiten von Kindern um, sie blieb damit aber hinter den Erwartungen der Bürger zurück. Zudem wurden die durchgeführten Maßnahmen schlecht vermarktet ${ }^{20}$.

Insgesamt gelang es der CDU jedoch besser als der SPD, die Wähler mit ihren Konzepten und ihren Leistungen zu überzeugen. Die strukturellen Vorteile, die sich aus der Verteilung der Parteiidentifikation ergaben, wurden durch die Themenorientierungen der Wählerschaft verstärkt. In dieser Konstellation hätte die SPD nur dann eine Chance zum Wahlsieg gehabt, wenn ihre Spitzenkandidatin die zuvor beschriebenen Defizite ausgeglichen hätte. Dies war aber aus zweierlei Gründen unwahrscheinlich: Anders als im Wettbewerb mit dem langjährigen Amtsinhaber Erwin Teufel konnte sie in der Auseinandersetzung mit dem neuen Ministerpräsidenten nicht auf Abnutzungserscheinungen ihres Gegenspielers setzen. Zudem ist es für eine Oppositionskandidatin immer schwierig, sich gegen einen amtierenden Regierungschef durchzusetzen. Wie eine Bestandsaufnahme der Einstellungen der Wähler zu den Spitzenkandidaten der beiden großen Parteien zeigt, zog die CDU denn auch aus der Kandidatenbewertung größere Vorteile als die SPD. Dabei muss man allerdings zwischen den beiden Grunddimensionen der Kandidatenbewertung, der Einschätzung der Persönlichkeit und der Leistungen der Kandidaten, unterscheiden.

19 Vgl. Honza Griese, Wahlkampf im Wandel am Beispiel der baden-württembergischen SPD, in: Josef Schmid/ Honza Griese (Hrsg.), Wahlkampf in Baden-Württemberg. Organisationsformen, Strategien und Ergebnisse der Landtagswahl vom 25. März 2001, Opladen 2002, S. 99 - 113.

20 Vgl. Oscar W. Gabriel / Kerstin Völkl, a.a.O. 
In der Bekanntheit der beiden Spitzenkandidaten lag Günther Oettinger deutlich vor seiner Konkurrentin. Auf die Frage, wer bei der Landtagswahl Spitzenkandidat der CDU für das Amt des Ministerpräsidenten sei, waren zwei Drittel der Befragten in der Lage, die richtige Antwort zu geben. Damit erreichte Oettinger auf Anhieb den Wert, den sein langjähriger Amtsvorgänger Teufel fünf Jahre zuvor erzielt hatte. Das Ergebnis ist insofern nachvollziehbar, als er bereits 14 Jahre das Amt des Fraktionsvorsitzenden der badenwürttembergischen CDU bekleidet hatte und einem Großteil der Bürger durch seine frühere Tätigkeit bekannt gewesen sein dürfte. Die Spitzenkandidatin der SPD, Ute Vogt, kannten 57 Prozent der Bürger. Damit konnte sie ihren Bekanntheitsgrad gegenüber 2001 nur um drei Prozentpunkte steigern. Dies spiegelt vermutlich ihre von manchen Beobachtern konstatierte geringe Präsenz in der Landespolitik wider.

Bei der Frage nach dem präferierten Ministerpräsidenten lassen sich kaum Veränderungen zwischen den Landtagswahlen 2001 und 2006 feststellen. Knapp die Hälfte der Bürger bevorzugte den CDU-Kandidaten als Ministerpräsidenten, während 41 Prozent (2006) beziehungsweise 45 Prozent (2001) lieber Vogt als Regierungschefin gesehen hätten. Wie die von Infratest dimap erhobenen Daten zeigen, verfügte Oettinger aber im Gegensatz zu seinem Vorgänger Teufel in sämtlichen Umfragen über einen klaren Vorsprung vor seiner Konkurrentin. Diese war in der ersten Märzumfrage 2001 bis auf zwei Prozentpunkte an Teufel herangerückt. Zwischen Günther Oettinger und Ute Vogt lag dagegen in keiner Umfrage ein ähnlich knapper Abstand ${ }^{21}$.

Verglichen mit der allgemeinen Frage nach dem bevorzugten Ministerpräsidenten liefert eine Analyse der Eigenschaftsprofile genauere Erkenntnisse darüber, wie die Wähler das persönliche Erscheinungsbild und die politischen Kompetenzen der Spitzenkandidaten bewerten. Besonders interessant ist dieser Vergleich, weil Vogt in den beiden Wahlen zwei CDU-Politikern mit einem sehr unterschiedlichen Profil gegenüberstand. Wie die Gegenüberstellung der Bewertung von Persönlichkeit und Leistung der CDU- und SPDSpitzenkandidaten zeigt, wurden nicht nur Teufel und Oettinger von den Bürgern sehr unterschiedlich wahrgenommen, auch das Bild von Vogt änderte sich zwischen 2001 und 2006 (vgl. Tabelle 3).

Die hervorstechenden Eigenschaften des Ministerpräsidenten sahen die Befragten in seiner Qualität als Siegertyp, in seinem Sachverstand und seiner Tatkraft. Auf jeder dieser drei Eigenschaftsdimensionen wurde er besser bewertet als sein Amtsvorgänger und lag deutlich vor seiner sozialdemokratischen Konkurrentin. Dies hatte sich vor fünf Jahren noch anders dargestellt: Nur der Sachverstand Teufels war höher eingestuft worden als der des Neulings Vogt. Bei der Tatkraft und der Bewertung der Siegerqualitäten lagen beide Spitzenkandidaten 2001 ungefähr gleichauf. Bei drei wichtigen leistungsbezogenen Merkmalen war es Oettinger gelungen, Imageschwächen seines Vorgängers zu überwinden und sich den Wählern als die im Vergleich mit Vogt wesentlich kompetentere Führungsfigur darzustellen.

Dem standen drei Schwachpunkte gegenüber: Glaubwürdigkeit, Sympathie und Bürgernähe. Abgesehen von der Bürgernähe hatte Teufel fünf Jahre zuvor ähnliche Bewertungen erhalten wie sein Nachfolger. Die Bürgernähe jedoch war sein Markenzeichen, das

21 In der Januarumfrage von Infratest dimap, in der der Abstand am kleinsten war, betrug er immer noch sechs Prozentpunkte. 
ihn 2001 deutlich von seiner sozialdemokratischen Konkurrentin abgesetzt hatte und ihn auch von Oettinger unterschied. Während 59 Prozent der Befragten Teufel als bürgernah einstuften, ordneten nur 24 Prozent Oettinger dieses Attribut zu. Damit lag er klar hinter Vogt, deren Bewertung auf der Eigenschaftsdimension Bürgernähe sich von 11 auf 43 Prozentpunkte verbesserte. Die zweite große Stärke Vogts war 2001 und 2006 ihre Einschätzung als sympathisch. Dieses Image blieb stabil und hob sie in beiden Wahlkämpfen deutlich von dem der CDU-Kandidaten ab, die von den Wählern relativ ähnlich bewertet wurden.

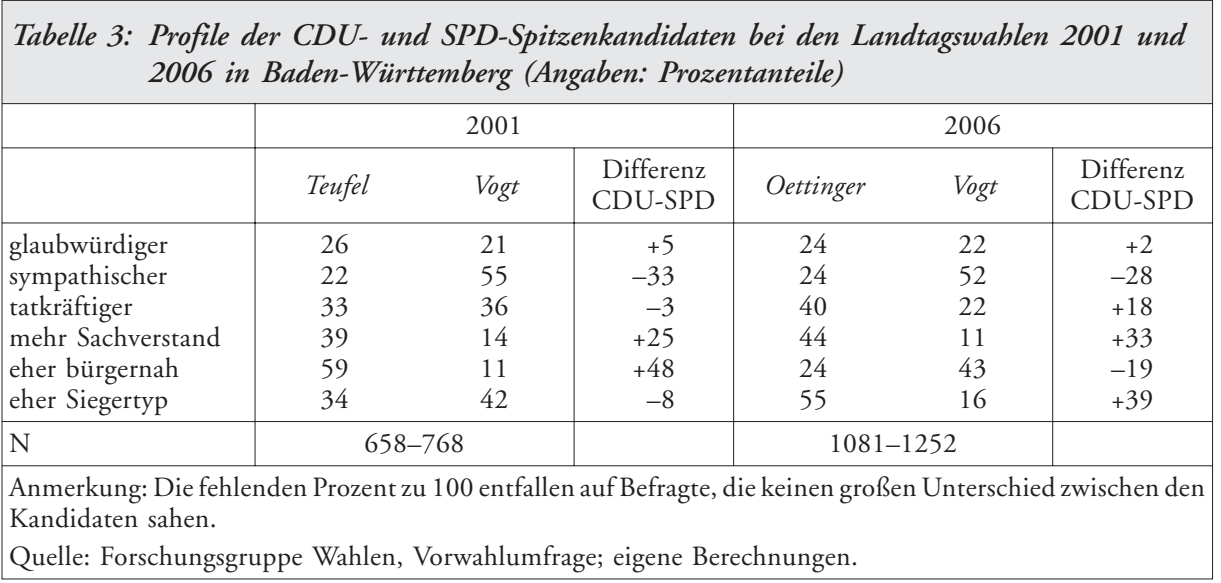

Sowohl 2001 als auch 2006 schätzten die Wähler die Kandidaten von CDU und SPD sehr unterschiedlich ein, wobei es in der Bewertung einige stabile und einige veränderliche Komponenten gab. Die Konstanten waren die Faktoren Sympathie und Kompetenz. Im ersten Bereich verzeichnete die SPD klare Vorteile, im zweiten die CDU. Abgesehen von der generell sehr kritisch bewerteten Glaubwürdigkeit veränderten sich bei allen anderen Imagekomponenten die Urteile über die Kandidaten - insbesondere wenn man die 2001 und 2006 bestehenden Konstellationen miteinander vergleicht. Da keiner der Kandidaten die Wähler auf allen Eigenschaftsdimensionen völlig überzeugte, aber auch keiner inakzeptabel erschien, befanden sie sich in einer schwierigen Entscheidungssituation. Nur für die Wählergruppen, die entweder der Sympathie oder der Leistung Priorität einräumten, war die Sachlage eindeutig. Für die erste Gruppe war Vogt die attraktivere Alternative, für die zweite Oettinger ${ }^{22}$. Stellt man einen Zusammenhang zwischen den Kandidatenprofilen und den Themen, die von den Bürgern im Vorfeld der Wahl als besonders wichtig erachtet wurden, her, dann dürften die Tatkraft und der Sachverstand Oettingers sowie sein siegessicheres Auftreten den Ausschlag dafür gegeben haben, dass er bei der Frage nach dem bevorzugten Ministerpräsident vor Vogt lag. Offensichtlich trugen so-

22 Vgl. Oscar W. Gabriel, Die baden-württembergische Landtagswahl vom 25. März 2001: Fehlschlag oder "Teufelsaustreibung", in: ZParl, 33. Jg. (2002), H. 1, S. $10-26$; ders. / S. Isabell Thaidigsmann / Kerstin Völkl, Alles bleibt so wie es war! Erklärungsfaktoren für die Wählerentscheidung bei der baden-württembergischen Landtagswahl 2001, in: Josef Schmid / Honza Griese (Hrsg.), a.a.O., S. 153 - 172. 
wohl die generelle Einschätzung der Spitzenkandidaten als auch die Bewertung ihrer spezifischen Eigenschaften zum Erfolg der CDU bei der Landtagswahl am 26. März 2006 bei.

In der Gesamtbilanz aller für die Wahlentscheidung bedeutsamen Faktoren war die CDU bei der Wahl zum Landtag 2006 deutlich im Vorteil gegenüber der SPD. Bei der Parteibindung lag sie klar vor den Sozialdemokraten, und auch bei den Themen- und Kandidatenorientierungen präsentierte sie sich den Wählern offenkundig als die attraktivere Alternative. Da diese drei Bestimmungsfaktoren jedoch nicht unabhängig voneinander auf die Wahlentscheidung der Bürger wirken, soll nunmehr untersucht werden, welchen Beitrag die Parteiidentifikation, die Problemlösungskompetenz der Parteien und die Kandidatenpräferenz in ihrem Zusammenspiel zum Erfolg der CDU beziehungsweise zum Misserfolg der SPD leisteten.

$\mathrm{Zu}$ diesem Zweck wurden Schätzmodelle berechnet, die den Einfluss der einzelnen Faktoren auf die Wahlentscheidung der Bürger zu Gunsten von CDU und SPD ausweisen und dadurch Rückschlüsse auf die Wahrscheinlichkeit einer CDU- beziehungsweise SPD-Wahl erlauben (tabellarisch nicht ausgewiesen). Den größten Einfluss der drei untersuchten Bestimmungsfaktoren übte sowohl im Falle einer CDU- als auch einer SPDWahl die langfristige Parteibindung aus. Die Kandidaten- und Themenorientierungen hatten bei Bürgern mit und ohne Parteibindung unterschiedliche Effekte. Für CDUWähler ohne Parteiidentifikation war die Kandidatenpräferenz wichtiger als die Problemlösungskompetenz, bei den SPD-Wählern verhielt es sich umgekehrt. Letztere machten ihre Wahlentscheidung zwar auch von der Bewertung der Spitzenkandidatin abhängig, wiesen der Problemlösungskompetenz der SPD jedoch größere Wichtigkeit zu. Die Parteiidentifizierer räumten den Kurzfristfaktoren ebenfalls unterschiedliche Bedeutung ein. Während für die CDU-Identifizierer die Präferenz für Oettinger als Ministerpräsidenten und die Lösungskompetenz der CDU auf dem Gebiet der Arbeitsmarktpolitik ungefähr gleich wichtig waren, entschieden die SPD-Wähler mit Parteiidentifikation in erster Linie kandidatenorientiert. Die modelltheoretisch geschätzte Dominanz der Parteiidentifikation bei der Wahlentscheidung findet ihre Entsprechung in der Selbsteinschätzung der Bürger. Auf die Frage, ob die Partei oder die Spitzenkandidaten für ihre persönliche Wahlentscheidung wichtiger seien, nannten 79 Prozent aller Befragten die Partei.

\section{Regierungsbildung und Oppositionsformierung}

Aus dem Wahlergebnis konnte die CDU unwidersprochen einen klaren Auftrag zur Regierungsbildung ableiten. Da sich die FDP bereits im Wahlkampf auf die Fortsetzung der bisherigen Regierungskoalition festgelegt hatte, war eine Regierungsbildung gegen die CDU, selbst wenn sie rechnerisch möglich war, politisch keine wirkliche Option. Schon am Wahlabend gestand die SPD denn auch ihre Wahlniederlage ein, während die CDU, die die absolute Mehrheit der Landtagsmandate nur um einen Sitz verfehlt hatte, die Führungsrolle in der neuen Landesregierung in Anspruch nahm und aus einer Position der Stärke heraus in die Koalitionsverhandlungen eintreten konnte.

Diese günstige Ausgangslage ergab sich auch daraus, dass sich der Ministerpräsident im Wahlkampf nicht auf eine Fortsetzung der CDU/FDP-Koalition festgelegt hatte. 
Folglich kündigte er bereits am Tag nach der Wahl an, Sondierungsgespräche mit der FDP und den Grünen führen zu wollen, ließ allerdings eine Präferenz für die Fortsetzung der bestehenden Koalition erkennen. Die CDU nahm die Gespräche mit beiden potenziellen Koalitionspartnern zügig auf, entschied sich jedoch nach nur zwei Runden dagegen, in offizielle Koalitionsverhandlungen mit den Grünen einzutreten. Der wichtigste Grund für das sehr schnelle Ende des schwarz-grünen Flirts ist wohl darin zu sehen, dass es keinen vernünftigen, der Öffentlichkeit vermittelbaren Grund dafür gab, die gut funktionierende und aus Sicht der CDU bequeme Zusammenarbeit mit der FDP zu beenden. Eine wichtige Rolle spielten aber auch das Störfeuer des konservativen Flügels der CDU und die Zurückhaltung der Bundesspitze der Grünen gegenüber dem schwarz-grünen Experiment. In Anbetracht der erst ein gutes Jahr zurückliegenden innerparteilichen Auseinandersetzung um die Teufel-Nachfolge, die in den Medien auch als Richtungsstreit zwischen Modernisierern und Konservativen gewertet worden war, dürfte Ministerpräsident Oettinger davor zurückgeschreckt sein, wegen eines Experiments mit ungewissem Ausgang eine Schwächung seiner Position zu riskieren.

Da sich weder in der Regierungsarbeit noch im Wahlkampf ernsthafte Konfliktlinien zwischen der CDU und der FDP gezeigt hatten, verliefen die Koalitionsverhandlungen komplikationslos. Am 9. Mai 2006 unterzeichneten die Spitzen beider Parteien die Koalitionsvereinbarung für die 14. Legislaturperiode. Die Schwerpunkte der künftigen Regierungsarbeit entsprachen den Erwartungen der Beobachter. Die Leitbotschaft des 80 Seiten starken Vertragswerkes lautete: „Baden-Württemberg steht vor großen Herausforderungen. Beispielhaft seien hier nur genannt: die Sicherung von Arbeitsplätzen und wirtschaftlichem Wachstum, die Bewältigung des demographischen Wandels, die Weiterentwicklung unseres Bildungssystems und der Betreuungsangebote für Kinder aller Altersstufen, die Integration von Zuwanderern und der Abbau des Haushaltsdefizits. "23

Priorität räumten die Koalitionspartner der Weiterentwicklung des Bildungssystems und der Betreuungsangebote für Kinder sowie dem Abbau des Haushaltsdefizits ein, wobei sie das ehrgeizige Ziel verfolgten, die Nettoneuverschuldung des Landes bis zum Ende der Legislaturperiode auf Null zu reduzieren ${ }^{24}$. Teil dieser Anstrengungen sollte eine systematische Aufgabenkritik sein, die als Basis einer Weiterentwicklung der bereits von der Regierung Teufel initiierten Verwaltungsreform dienen sollte.

Noch unspektakulärer als die Vereinbarungen über das Arbeitsprogramm fielen die Ergebnisse der Verhandlungen über die Zusammensetzung der künftigen Landesregierung aus. Dies war insofern nicht erstaunlich, als die Ministerpräsidenten Teufel und Oettinger bereits in den Jahren unmittelbar vor der Landtagswahl 2006 umfassende personelle Veränderungen vorgenommen hatten. Lediglich vier der nach der Landtagswahl 2001 bestellten Kabinettsmitglieder gehörten auch der zweiten Regierung Oettinger an, zwei davon in einem neuen Ressort. Nach dem Wechsel im Amt des Ministerpräsidenten hatte Oettinger bereits vier neue Minister ernannt: Heribert Rech übernahm das Innenministerium, Peter Hauk das Ministerium für Landwirtschaft und ländlichen Raum und An-

23 Vereinbarung zwischen der Christlich Demokratischen Union Deutschlands, Landesverband Baden-Württemberg, und der Freien Demokratischen Partei, Landesverband Baden-Württemberg, über die Bildung einer Koalitionsregierung für die 14. Legislaturperiode des Landtags von Baden-Württemberg, S. 2.

24 Ebenda, S. 59. 
dreas Renner das Sozialministerium. Wolfgang Reinhard wurde Bevollmächtigter des Landes Baden-Württemberg beim Bund. Die bisherige Sozialministerin Tanja Gönner wechselte ins Umweltressort. Weitere Veränderungen des Personaltableaus folgten. Nach der Bundestagswahl 2005 wurde die bisherige Kultusministerin Schavan Bundesministerin und wurde durch den bisherigen Staatssekretär Helmut Rau ersetzt. Kurz vor Beginn des heißen Wahlkampfes musste Sozialminister Renner wegen eines Konflikts mit dem Bischof von Stuttgart und Rottenburg seinen Rücktritt einreichen. An seine Stelle trat die Staatssekretärin im Kultusministerium Monika Stolz. So erfolgten die einzigen personellen Änderungen auf nachrangigen Positionen (vgl. Tabelle 4). Einen Einschnitt signalisierte lediglich die für 2008 angekündigte Auswechslung von Finanzminister Gerhard Stratthaus.

Tabelle 4: Zusammensetzung des Ministerrates von Baden-Württemberg (Stand: 2006)

\begin{tabular}{|c|c|c|}
\hline & $\mathrm{alt}^{*}$ & neu \\
\hline Ministerpräsident & \multicolumn{2}{|c|}{ Günther Oettinger (CDU) } \\
\hline $\begin{array}{l}\text { Justizminister und stellvertretender Ministerpräsident, } \\
\text { Integrationsbeauftragter des Landes }\end{array}$ & \multicolumn{2}{|c|}{ Prof. Dr. Ulrich Goll (FDP) } \\
\hline $\begin{array}{l}\text { Minister des Staatsministeriums und für europäische } \\
\text { Angelegenheiten }\end{array}$ & \multicolumn{2}{|c|}{ Willi Stächele (CDU) } \\
\hline Innenminister & \multicolumn{2}{|c|}{ Heribert Rech (CDU) } \\
\hline Minister für Kultus, Jugend und Sport & \multicolumn{2}{|c|}{ Helmut Rau (CDU) } \\
\hline Minister für Wissenschaft, Forschung und Kunst & \multicolumn{2}{|c|}{ Prof. Dr. Peter Frankenberg (CDU) } \\
\hline Wirtschaftsminister & \multicolumn{2}{|c|}{ Ernst Pfister (FDP) } \\
\hline Finanzminister & \multicolumn{2}{|c|}{ Gerhard Stratthaus (CDU) } \\
\hline Minister für Ernährung und Ländlichen Raum & \multicolumn{2}{|c|}{ Peter Hauk (CDU) } \\
\hline $\begin{array}{l}\text { Ministerin für Arbeit und Soziales, Kinderbeauftragte } \\
\text { der Landesregierung, Beauftragte der Landesregierung } \\
\text { für Chancengleichheit von Frauen und Männern }\end{array}$ & $\begin{array}{l}\text { Andreas Renner } \\
\quad(\mathrm{CDU})\end{array}$ & $\begin{array}{l}\text { Dr. Monika Stolz } \\
\text { (CDU) }\end{array}$ \\
\hline Umweltministerin & \multicolumn{2}{|c|}{ Tanja Gönner (CDU) } \\
\hline $\begin{array}{l}\text { Minister und Bevollmächtigter des Landes } \\
\text { Baden-Württemberg beim Bund }\end{array}$ & \multicolumn{2}{|c|}{ Prof. Dr. Wolfgang Reinhard (CDU) } \\
\hline $\begin{array}{l}\text { Staatssekretär im Wirtschaftsministerium mit } \\
\text { Stimmrecht in der Regierung }\end{array}$ & $\begin{array}{l}\text { Dr. Horst Mehrländer } \\
\text { (FDP) }\end{array}$ & $\begin{array}{l}\text { Richard Drautz } \\
\quad \text { (FDP) }\end{array}$ \\
\hline $\begin{array}{l}\text { Ehrenamtliche Staatsrätin für demographischen Wandel } \\
\text { und Senioren im Staatsministerium }\end{array}$ & & $\begin{array}{l}\text { Prof. Dr. Claudia } \\
\text { Hübner (CDU) }\end{array}$ \\
\hline
\end{tabular}

* Die Zusammensetzung des „alten“ Ministerrates bezieht sich auf den Stand vom 26. April 2005, als Günther Oettinger kurz nach seiner Wahl zum Ministerpräsidenten als Nachfolger von Erwin Teufel sein neues Kabinett vorstellte. Im „neuen“ Ministerrat nach der Landtagswahl 2006 wurde das Amt des ehrenamtlichen Staatsrats für Lebenswissenschaften im Staatsministerium, das Konrad Beyreuther innehatte, in das für demographischen Wandel und Senioren im Staatsministerium umgewidmet. Ebenso wurde das Amt des stellvertretenden Ministerpräsidenten vom Wirtschaftsminister Ernst Pfister (FDP) auf den Justizminister Ulrich Goll (FDP) übertragen, der außerdem die Aufgabe des Integrationsbeauftragten zugewiesen bekam. Auch das Ministerium für Arbeit und Soziales wurde um die Bereiche Kinder und Chancengleichheit von Frauen und Männern erweitert.

Quelle: Ministerrat alt: http://www.lpb.bwue.de/ministerpraesident/ministerpraesident.php3; Ministerrat neu: http://www.baden-wuerttemberg.de/de/ministerrat/86052.html. 
Am 14. Juni 2006 stellte sich Ministerpräsident Oettinger im Landtag erfolgreich zur Wiederwahl. Er erhielt nicht nur alle Stimmen der Koalitionsfraktionen, sondern wurde auch von zwei Abgeordneten der Opposition gewählt. Die neue Landesregierung wurde am selben Tag vereidigt und nahm ihre Arbeit auf.

Ute Vogt wurde zur Vorsitzenden der SPD-Fraktion gewählt, den Landesparteivorsitz hat sie bereits seit 1999 inne. Der bisherige Fraktionsvorsitzende Wolfgang Drexler amtiert nun als erster stellvertretender Landtagspräsident. Die Fraktion der Grünen führt weiterhin Winfried Kretschmann.

\section{Zusammenfassung}

(1) Wie bei allen seit der Gründung des Landes durchgeführten Landtagswahlen konnte sich die CDU auch am 26. März 2006 als stärkste politische Kraft in Baden-Württemberg behaupten. Der Wahlsieg fiel noch klarer aus als fünf Jahre zuvor, als die SPD mit ihrer neuen Spitzenkandidatin Ute Vogt einen Achtungserfolg erzielt und die 30-Prozent-Marke übertroffen hatte. Die CDU konnte zwar nicht an die guten Ergebnisse der 1970er und 1980er Jahre anknüpfen, stabilisierte sich aber auf dem Niveau (44,2 Prozent), das sie in der letzten Amtszeit Erwin Teufels erreicht hatte.

(2) Für die SPD entwickelte sich die Landtagswahl 2006 (25,2 Prozent) dagegen zu einem in seinem Ausmaß nicht unbedingt erwarteten Fiasko; nur 1996 hatte die Partei noch schlechter abgeschnitten.

(3) Die beiden kleinen Parteien erzielten sehr gute Ergebnisse (Grüne: 11,7 Prozent; FDP: 10,7 Prozent). Sie profitierten unter anderem von dem Umstand, dass Wähler, die der CDU kritisch gegenüberstanden, teilweise für die Grünen oder die FDP votierten - vorausgesetzt sie enthielten sich nicht ihrer Stimme.

(4) Die Wahlbeteiligung fiel mit 53,4 Prozent so niedrig aus wie noch nie zuvor bei einer Landtagswahl in diesem Bundesland. Das heißt, dass nur 23,3 Prozent der Wahlberechtigten (nicht der Wähler) für die CDU stimmten und nur 13,2 Prozent für die SPD.

(5) Maßgeblich für den Ausgang des Rennens zwischen der CDU und der SPD war zunächst die Verteilung der Parteiidentifikation. Sie schuf die Grundlage für den Fortbestand der CDU-Dominanz in einem Land, in dem auf Grund seiner Sozialstruktur CDU-Erfolge nicht vorprogrammiert sind.

(6) Bei der Landtagswahl 2006 kam aber nicht nur die Verteilung der langfristigen Parteiidentifikation zum Tragen; vielmehr profitierte die CDU auch von den Themenund Kandidatenorientierungen. In keinem für die Wahlentscheidung relevanten Bereich hatte die SPD der für die CDU günstigen Stimmungslage etwas entgegenzusetzen.

(7) Insgesamt blieben der Wahlkampf, der Wahlausgang und die Regierungsbildung frei von Überraschungen. Auch eine Weichenstellung für eine schwarz-grüne Zusammenarbeit jenseits der Kommunalebene blieb aus. 